\title{
FREQUENCY ANALYSIS OF PRECONDITIONED WAVEFORM RELAXATION ITERATIONS
}

Abstract. The error analysis of preconditioned waveform relaxation iterations for differential systems is presented. This analysis extends and refines previous results by Burrage, Jackiewicz, Nørsett and Renaut by incorporating all terms in the expansion of the error of waveform relaxation iterations in the Laplace transform domain. Lower bounds for the size of the window of rapid convergence are also obtained. The theory is illustrated for waveform relaxation methods applied to differential systems resulting from semi-discretization of the heat equation in one and two dimensions. This theory and some heuristic arguments predict that preconditioning is most effective for the first few iterations.

1. Introduction. Consider the linear system of differential equations

$$
\left\{\begin{array}{l}
u^{\prime}(t)+Q u(t)=f(t), \quad t \in[0, T] \\
u(0)=u_{0}
\end{array}\right.
$$

with a constant matrix $Q$ of dimension $N \times N$. To solve (1.1) the matrix $Q$ is split as

$$
Q=M+D
$$

and we define iterations of the form

1991 Mathematics Subject Classification: Primary 65L05.

Key words and phrases: waveform relaxation, splitting, preconditioning, convergence, error analysis.

The work of A. Augustynowicz was partially supported by University of Gdańsk under grant BW 5100-5-0052-6.

The work of Z. Jackiewicz was partially supported by the National Science Foundation under grant NSF DMS-9208048. 


$$
\left\{\begin{array}{l}
\frac{d}{d t} u^{(k+1)}(t)+M u^{(k+1)}(t)=f(t)-D u^{(k)}(t), \quad t \in[0, T], \\
u^{(k+1)}(0)=u_{0},
\end{array}\right.
$$

$k=0,1, \ldots$, where $u^{(0)}$ is a given initial guess. These waveform relaxation iterations were first proposed by Lelarasmee et al. [5] and were extensively studied in the last few years by Miekkala and Nevanlinna [6], Nevanlinna [7], Leimkuhler [4], Burrage et al. [2], [3] and others. We refer to the recent book by Burrage [1] for a good introduction to this topic and for a review of the current literature in this area.

It is well known that the iterations (1.3) converge superlinearly on any interval and for any splitting of the matrix $Q$ (see [6]). However, the convergence is often slow for (1.3) to be useful for practical purposes and it is of interest to accelerate the speed of this convergence. One of the techniques to accomplish this goal is the exponential preconditioning proposed in [2]. The idea behind this technique is the following. If we split the matrix $Q$ as in (1.2) and make the transformation

$$
w(t)=e^{t D} u(t),
$$

then the equation (1.1) can be rewritten in the form

$$
\left\{\begin{array}{l}
w^{\prime}(t)+B(t) w(t)=e^{t D} f(t), \quad t \in[0, T], \\
w(0)=u_{0},
\end{array}\right.
$$

where $B(t)=e^{t D} M e^{-t D}$. Furthermore, the splitting $B(t)=M-N(t)$ leads to the iterations

$$
\left\{\begin{array}{l}
\frac{d}{d t} w^{(k+1)}(t)+M w^{(k+1)}(t)=N(t) w^{(k)}(t)+e^{t D} f(t), \quad t \in[0, T] \\
w^{(k+1)}(0)=u_{0}
\end{array}\right.
$$

which may converge faster than the iterations (1.3) if the matrix $M$ is chosen in a proper way.

Denote the error of $w^{(k)}$ by $\varepsilon^{(k)}$. Then subtracting (1.4) from (1.5) we obtain the error equation

$$
\left\{\begin{array}{l}
\frac{d}{d t} \varepsilon^{(k+1)}(t)+M \varepsilon^{(k+1)}(t)=N(t) \varepsilon^{(k)}(t), \quad t \in[0, T], \\
\varepsilon^{(k+1)}(0)=0
\end{array}\right.
$$

$k=0,1, \ldots$ Define the operator

$$
\mathcal{K}_{P} u(t)=\int_{0}^{t} e^{(s-t) M} N(s) d s
$$

and denote by $\varrho_{\alpha}(\cdot)$ the spectral radius in the space of functions with the weighted norm 


$$
\|y\|_{\alpha}:=\sup \left\{e^{-\alpha t}|y(t)|: t \in[0, \infty)\right\}<\infty .
$$

Then

$$
\varepsilon^{(k+1)}(t)=\mathcal{K}_{P} \varepsilon^{(k)}(t)
$$

and it follows from the results of Miekkala and Nevanlinna [6] that

$$
\varrho_{\alpha}\left(\mathcal{K}_{P}\right)=\sup \left\{\varrho\left(K_{P}(s)\right): \operatorname{Re}(s)=\alpha\right\},
$$

where $\varrho\left(K_{P}(s)\right)$ is the spectral radius of a matrix $K_{P}(s)$ which may be interpreted as the Laplace transform of the kernel of the operator $\mathcal{K}_{P}$. In the next section we will use $K_{P}(s)$ and (1.7) to estimate the size of the window of convergence of the waveform relaxation iterations (1.5).

2. Error analysis in Laplace transform domain. It was demonstrated in [2] that the matrix $N(t)$ has the expansion

$$
N(t)=M-\sum_{i=0}^{\infty} \frac{t^{i}}{i !} \Delta_{i}, \quad \text { where } \quad \Delta_{i}=\sum_{j=0}^{i}\left(\begin{array}{l}
i \\
j
\end{array}\right) D^{i-j} M(-D)^{j}
$$

and the matrices $\Delta_{i}$ are related by the recurrence relation

$$
\Delta_{i+1}=D \Delta_{i}-\Delta_{i} D, \quad i=0,1, \ldots, \quad \Delta_{0}=M .
$$

The equation (1.6) now takes the form

$$
\left\{\begin{array}{l}
\frac{d}{d t} \varepsilon^{(k+1)}(t)+M \varepsilon^{(k+1)}(t)=-\sum_{i=1}^{\infty} \frac{t^{i}}{i !} \Delta_{i} \varepsilon^{(k)}(t), \quad t \in[0, T], \\
\varepsilon^{(k+1)}(0)=0,
\end{array}\right.
$$

$k=0,1, \ldots$, and applying the Laplace transform we get

$$
(s I+M) \widehat{\varepsilon}^{(k+1)}(s)=\sum_{i=1}^{\infty} \frac{(-1)^{i+1}}{i !} \Delta_{i} \frac{d^{i}}{d s^{i}} \widehat{\varepsilon}^{(k)}(s) .
$$

Here, $I$ is the identity matrix of dimension $N$.

In [2] the relation (2.2) was analyzed by taking into account only the term corresponding to $\Delta_{1}$ and assuming that $\varepsilon^{(0)}(t)=t v$ for some fixed vector $v \in \mathbb{R}^{N}$. In this paper we will take into account all terms in the expression on the right hand side of (2.2) and consider a more general initial error of the form $\varepsilon^{(0)}(t)=t^{r} v$. Under these assumptions the equation (2.2) for $k=0$ takes the form

$$
\widehat{\varepsilon}^{(1)}(s)=K_{P, r}(s) \widehat{\varepsilon}^{(0)}(s),
$$

where

$$
K_{P, r}(s)=\sum_{i=1}^{\infty} \frac{(i+r) !}{i ! r !} s^{-i}(s I+M)^{-1} \Delta_{i}
$$


Taking into account only the first term in (2.3) we obtain the matrix

$$
K_{P, r}^{1}(s)=\frac{r+1}{s}(s I+M)^{-1} \Delta_{1},
$$

which was used in [2] for $r=1$ as an approximation to $K_{P}(s)$.

We will employ (2.3) to investigate the speed of convergence of the corresponding waveform relaxation iterations (1.5). We will illustrate first this approach for the linear system obtained from semidiscretization of the heat equation in one space variable. This system takes the form

$$
\left\{\begin{array}{l}
y^{\prime}(t)+Q y(t)=0, \quad t>0 \\
y(0)=y_{0}
\end{array}\right.
$$

where $y \in \mathbb{R}^{N}$, and

$$
Q=\left[\begin{array}{ccccc}
2 & -1 & & & \\
-1 & 2 & -1 & & \\
& \ddots & \ddots & \ddots & \\
& & -1 & 2 & -1 \\
& & & -1 & 2
\end{array}\right] \in \mathbb{R}^{N \times N} .
$$

We assume that $N=b n$ for some positive integers $b$ and $n$ and consider the splitting (1.2) with the block diagonal matrix $M$ corresponding to the Gauss-Jacobi method with block size $b$. It is then possible to obtain closed form expressions for $K_{P, r}(s)$ given by (2.3). This matrix is given by

$$
\begin{aligned}
K_{P, r}(s)= & \frac{1}{2}(s I+M)^{-1}\left\{\left(\left(\frac{s}{s-1}\right)^{r+1}-\left(\frac{s}{s+1}\right)^{r+1}\right) \Delta_{1}\right. \\
& \left.+2\left(\left(\frac{s}{s-1}\right)^{r+1}+\left(\frac{s}{s+1}\right)^{r+1}-2\right) \Delta_{2}\right\}
\end{aligned}
$$

for $b \geq 3$ and

$$
\begin{aligned}
K_{P, r}(s)= & \frac{1}{4}(s I+M)^{-1}\left\{\left(\left(\frac{s}{s-2}\right)^{r+1}-\left(\frac{s}{s+2}\right)^{r+1}\right)\left(\Delta_{1}+\Gamma_{1}\right)\right. \\
& +2\left(\left(\frac{s}{s+1}\right)^{r+1}-\left(\frac{s}{s-1}\right)^{r+1}\right) \Gamma_{1} \\
& +\frac{1}{2}\left(\left(\frac{s}{s-2}\right)^{r+1}+\left(\frac{s}{s+2}\right)^{r+1}-2\right)\left(\Delta_{2}+\Gamma_{2}\right) \\
& \left.+2\left(2-\left(\frac{s}{s-1}\right)^{r+1}-\left(\frac{s}{s+1}\right)^{r+1}\right) \Gamma_{2}\right\}
\end{aligned}
$$


for $b=2$, where $\Gamma_{1}$ and $\Gamma_{2}$ are $N \times N$ matrices with

$$
\begin{aligned}
& \left(\Gamma_{1}\right)_{i j}= \begin{cases}1 & \text { if }(i, j) \in\{(1,3),(N, N-2)\}, \\
-1 & \text { if }(i, j) \in\{(3,1),(N-2, N)\}, \\
0 & \text { otherwise }\end{cases} \\
& \left(\Gamma_{2}\right)_{i j}= \begin{cases}1 & \text { if }(i, j) \in\{(1,2),(2,1),(N-1, N),(N, N-1)\}, \\
0 & \text { otherwise. }\end{cases}
\end{aligned}
$$

To justify these expressions for $K_{P, r}(s)$ observe that

$$
\begin{aligned}
D & =\left[\begin{array}{ccccc}
0 & \widehat{D} & & & \\
\widehat{D}^{T} & 0 & \widehat{D} & & \\
& \ddots & \ddots & \ddots & \\
& & \widehat{D}^{T} & 0 & \widehat{D} \\
& & \widehat{D}^{T} & 0
\end{array}\right] \in \mathbb{R}^{N \times N}, \\
\Delta_{1} & =\left[\begin{array}{ccccc}
0 & \widehat{\Delta}_{1} & & & \\
-\widehat{\Delta}_{1}^{T} & 0 & \widehat{\Delta}_{1} & & \\
& \ddots & \ddots & \ddots & \\
& & -\widehat{\Delta}_{1}^{T} & 0 & \widehat{\Delta}_{1} \\
& & & -\widehat{\Delta}_{1}^{T} & 0
\end{array}\right] \in \mathbb{R}^{N \times N},
\end{aligned}
$$

where $\widehat{D}$ and $\widehat{\Delta}_{1}$ are $b \times b$ matrices defined by

$$
\begin{aligned}
(\widehat{D})_{i j} & = \begin{cases}-1 & \text { if }(i, j)=(b, 1), \\
0 & \text { otherwise }\end{cases} \\
\left(\widehat{\Delta}_{1}\right)_{i j} & = \begin{cases}-1 & \text { if }(i, j)=(b-1,1), \\
1 & \text { if }(i, j)=(b, 2), \\
0 & \text { otherwise. }\end{cases}
\end{aligned}
$$

These expressions for $\widehat{D}$ and $\widehat{\Delta}_{1}$ and $b \geq 3$ imply that

$$
\Delta_{3}=\Delta_{1}, \quad \Delta_{i+2}=\Delta_{i}, \quad i \geq 1 .
$$

hence,

$$
\begin{aligned}
(s I+M) K_{P, r}(s) & =\frac{s^{r+1}}{r !} \sum_{i=1}^{\infty} \frac{(i+r) !}{i !} s^{-(i+r+1)} \Delta_{i} \\
& =(-1)^{r} \frac{s^{r+1}}{r !} \frac{d^{r}}{d s^{r}} \sum_{i=1}^{\infty} s^{-i-1} \Delta_{i} \\
& =(-1)^{r} \frac{s^{r+1}}{r !} \frac{d^{r}}{d s^{r}}\left(\sum_{j=1}^{\infty} s^{-2 j} \Delta_{1}+\sum_{j=1}^{\infty} s^{-2 j-1} \Delta_{2}\right) \\
& =(-1)^{r} \frac{s^{r+1}}{r !} \frac{d^{r}}{d s^{r}}\left(\frac{1}{s^{2}-1} \Delta_{1}+\frac{1}{s\left(s^{2}-1\right)} \Delta_{2}\right)
\end{aligned}
$$




$$
\begin{aligned}
= & \frac{1}{2}(-1)^{r} \frac{s^{r+1}}{2 r !} \frac{d^{r}}{d s^{r}}\left\{\left(\frac{1}{s-1}-\frac{1}{s+1}\right) \Delta_{1}\right. \\
& \left.+\left(\frac{1}{s-1}+\frac{1}{s+1}-\frac{2}{s}\right) \Delta_{2}\right\} \\
= & \frac{1}{2} s^{r+1}\left\{\left(\frac{1}{(s-1)^{r+1}}-\frac{1}{(s+1)^{r+1}}\right) \Delta_{1}\right. \\
& \left.+\left(\frac{1}{(s-1)^{r+1}}+\frac{1}{(s+1)^{r+1}}-\frac{2}{s^{r+1}}\right) \Delta_{2}\right\},
\end{aligned}
$$

which proves (2.6). If $b=2$ then it can be verified that

$$
\Delta_{2 i-1}=2^{2 i-2}\left(\Delta_{1}+\Gamma_{1}\right)-\Gamma_{1}, \quad \Delta_{2 i}=2^{2 i-2}\left(\Delta_{2}+\Gamma_{2}\right)-\Gamma_{2}
$$

for $i \geq 1$. Calculations similar to those for $b \geq 3$ prove (2.7).

Using heuristic arguments Leimkuhler [4] argued (compare also [2]) that the preconditioned waveform relaxation iterations (1.5) with initial error of the form $\varepsilon^{(0)}(t)=t^{r} v$ are convergent on the window approximately equal to $[0, \widehat{T}]$ where $\widehat{T}=1 / \operatorname{Re}(\widehat{s})$ and where $\widehat{s}$ is such that

$$
\varrho\left(K_{P, r}(s)\right)<1 \quad \text { for } \operatorname{Re}(s)>1 / \operatorname{Re}(\widehat{s}) .
$$

Using pseudospectra of waveform relaxation operators Jackiewicz, Owren and Welfert [3] demonstrated that a more accurate estimate of the window of convergence is the interval $[0, \widehat{T}]$, where $\widehat{T}=4 / \operatorname{Re}(\widehat{s})$. We have plotted in Fig. 1 the graphs of

$$
\sup \left\{\varrho\left(K_{P, r}(s)\right): \operatorname{Re}(s)=x\right\}
$$

against $x$ for $r=1$ and $r=4$ (dashed lines). For comparison we have also plotted the graphs of

$$
\sup \left\{\varrho\left(K_{P, r}^{1}(s)\right): \operatorname{Re}(s)=x\right\}
$$

for $r=1$ and $r=4$ (dashed-dotted lines), where $K_{P, r}^{1}(s)$ is defined by (2.4), and the graph of

$$
\sup \left\{\varrho\left(K_{N}(s)\right): \operatorname{Re}(s)=x\right\}
$$

(solid line), where $K_{N}(s)$ is the Laplace transform of the operator corresponding to the waveform relaxation iterations without preconditioning defined by (1.3). All these graphs correspond to $N=64$ and $b=32$.

We can see from this figure that using $\varrho\left(K_{P, r}(s)\right)$ we obtain a different and more accurate prediction about the size of the window of convergence of the iterations (1.5) than that obtained by using $\varrho\left(K_{P, r}^{1}(s)\right)$ as was done in [2]. For example we should expect that (1.5) with $\varepsilon^{(0)}(t)=t v$ will converge on the window approximately equal to $[0,2.8]$ if we use $\varrho\left(K_{P, r}(s)\right)$ and on the window approximately equal to $[0,4.4]$ if we use $\varrho\left(K_{P, r}^{1}(s)\right)$. The latter 
estimate is too optimistic and the numerical experiments presented in [2] confirm that the new estimate is more accurate. Similarly, we can predict using $\varrho\left(K_{P, r}(s)\right)$ that (1.5) should converge faster than (1.3) on the window approximately equal to [0,1.72]. This corresponds to $4 / x$ where $x \approx 2.3$ is the intersection of the solid and dashed curves in Fig. 1 corresponding to $K_{N}(s)$ and $K_{P, r}(s)$. Using $K_{P, r}^{1}(s)$ we get the estimate $[0,2]$ of this window. The numerical results given in [2] confirm again that the former estimate is more accurate.

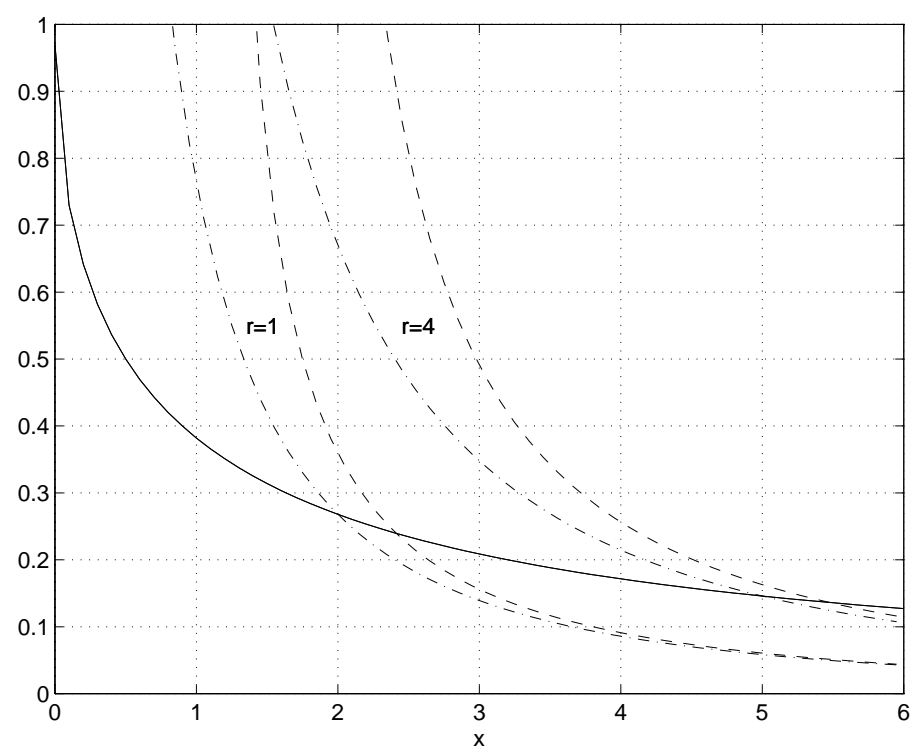

Fig. 1. Spectral radii corresponding to the one-dimensional heat equation. Dashed lines: $\sup \left\{\varrho\left(K_{P, r}(s)\right): \operatorname{Re}(s)=x\right\}$; dashed-dotted lines: $\sup \left\{\varrho\left(K_{P, r}^{1}(s)\right): \operatorname{Re}(s)=x\right\}$; solid line: $\sup \left\{\varrho\left(K_{N}(s)\right): \operatorname{Re}(s)=x\right\}$.

Since $\left\|\Delta_{i}\right\| \leq \sum_{j=0}^{i}\left(\begin{array}{c}i \\ j\end{array}\right)\|D\|^{i}\|M\|=\|M\| \cdot\|2 D\|^{i}$, we have

$$
\begin{aligned}
\left\|K_{P, k}(s)\right\| & \leq \sum_{i=1}^{\infty} \frac{(i+k) !}{i ! k !}|s|^{-i}\left\|(s I+M)^{-1}\right\| \cdot\|M\| \cdot\|2 D\|^{i} \\
& =\|M\| \cdot\left\|(s I+M)^{-1}\right\| \frac{(k+1) s^{k}}{(s-\|2 D\|)^{k+1}}=O\left(s^{-2}\right) .
\end{aligned}
$$

This estimate shows that $\widehat{\varepsilon}^{k}(s)=O\left(s^{-q-2 k}\right)$ if $\widehat{\varepsilon}^{(0)}(s)=O\left(s^{-q}\right)$. However, the coefficient $M(k)$ in $\left\|\widehat{\varepsilon}^{(k)}(s)\right\| \leq M(k)|s|^{-q-2 k}$ tends to infinity as $k \rightarrow \infty$. This suggests that for a small but fixed interval $[0, T]$ the preconditioning is the most effective for the first few iterations. The numerical experiments presented in Fig. 1 seem to confirm this suggestion. 
3. Spectral radius estimation. To graph $\sup \{\varrho(K(s)): \operatorname{Re}(s)=x\}$ as a function of $x$, where $K(s)=K_{P, r}(s)$ or $K(s)=K_{P, r}^{1}(s)$ are given by (2.3) or (2.4), respectively, it is important to know where the function $y \mapsto \varrho(K(x+i y))$ attains its maximum for fixed $x$. There are important examples where this maximum is reached at $y=0$ (compare [6]), but this is not always the case. In what follows we will estimate the size of the interval where this maximum is attained. This information is important from practical point of view since to find the maximum of $\varrho(K(x+i y))$ for fixed $x$ we need only search the corresponding compact interval.

Suppose that

$$
\varrho\left(K\left(x+i y_{m}\right)\right)=\sup \{\varrho(K(x+i y)): y \in \mathbb{R}\} .
$$

Assume first that $\lambda$ is an eigenvalue of $K_{P, r}^{1}(s)$ given by (2.4) and that $u$ is the corresponding eigenvector. Then

$$
2 \Delta_{1} u=\lambda s(s I+M) u
$$

and it follows that

$$
2 \delta_{1}=\lambda s(s+m), \quad \text { where } \quad \delta_{1}=\frac{u^{*} \Delta_{1} u}{u^{*} u}, \quad m=\frac{u^{*} M u}{u^{*} u}>0,
$$

and $u^{*}$ is the complex conjugate of the vector $u$. Let $s=x+i y, x>0$. Then

$$
|\lambda|<\frac{2\left|\delta_{1}\right|}{|s|^{2}}=\frac{2\left|\delta_{1}\right|}{x^{2}+y^{2}}
$$

and this implies that $\varrho\left(K_{P, r}^{1}(x+i y)\right) \leq \varrho\left(K_{P, r}^{1}(x)\right)$ for

$$
y^{2} \geq \frac{2\left|\delta_{1}\right|}{\varrho\left(K_{P, r}^{1}(x)\right)}-x^{2} .
$$

Since $\varrho\left(K_{P, r}^{1}(\bar{s})\right)=\varrho\left(K_{P, r}^{1}(s)\right)$ and

$$
\left|\delta_{1}\right| \leq \begin{cases}2 & \text { for } b=2,3 \\ 1 & \text { for } b \geq 4\end{cases}
$$

we obtain

$$
0 \leq y_{m} \leq \sqrt{\frac{a}{\varrho\left(K_{P, 1}^{1}(x)\right)}-x^{2}},
$$

where

$$
a= \begin{cases}4 & \text { for } b=2,3 \\ 2 & \text { for } b \geq 4 .\end{cases}
$$

Assume next that $b \geq 3$ and consider the matrix $K_{P, 1}(s)$ given by (2.3). The formula (2.6) yields 


$$
K_{P, 1}(s)=(s I+M)^{-1}\left(\frac{2 s^{3}}{\left(s^{2}-1\right)^{2}} \Delta_{1}+\frac{3 s^{2}-1}{\left(s^{2}-1\right)^{2}} \Delta_{2}\right) .
$$

Assuming that $K_{P, 1}(s) u=\lambda u$ we obtain

$$
\lambda(s+m)=\frac{2 s^{3}}{\left(s^{2}-1\right)^{2}} \delta_{1}+\frac{3 s^{2}-1}{\left(s^{2}-1\right)^{2}} \delta_{2},
$$

where $\delta_{1}$ and $m$ are defined as before and $\delta_{2}$ is given by

$$
\delta_{2}=\frac{u^{*} \Delta_{2} u}{u^{*} u} .
$$

For $x>1$ and $a \geq 2\left|\delta_{1}\right|+3\left|\delta_{2}\right|$ we can bound $|\lambda|$ as follows:

$$
\begin{aligned}
|\lambda| & <\frac{2|s|^{2}\left|\delta_{1}\right|+3|s| \cdot\left|\delta_{2}\right|}{\left|s^{2}-1\right|^{2}} \leq \frac{\left(2\left|\delta_{1}\right|+3\left|\delta_{2}\right|\right)|s|^{2}}{\left|s^{2}-1\right|^{2}} \\
& \leq \frac{a|s|^{2}}{|s-1|^{2}|s+1|^{2}}=\frac{a\left(x^{2}+y^{2}\right)}{\left(x^{2}-1\right)^{2}+2\left(x^{2}+1\right) y^{2}+y^{4}} .
\end{aligned}
$$

If we put

$$
\eta=\frac{a}{\varrho\left(K_{P, 1}(x)\right)},
$$

then the inequality $|\lambda| \geq \varrho\left(K_{P, 1}(x)\right)$ takes the form

$$
y^{4}+\left(2 x^{2}+2-\eta\right) y^{2}+\left(x^{2}-1\right)^{2}-\eta x^{2} \geq 0,
$$

which is equivalent to

$$
y^{2} \geq \sqrt{4 x^{2}-\eta+\eta^{2} / 4}-x^{2}-1+\eta / 2 .
$$

Since $a \geq 2\left|\delta_{1}\right|+3\left|\delta_{2}\right|$ and

$$
\left|\delta_{2}\right| \leq \begin{cases}2 & \text { for } b=3 \\ 1 & \text { for } b \geq 4,\end{cases}
$$

we can conclude that $\varrho\left(K_{P, 1}(x+i y)\right)$ attains its maximum for fixed $x>1$ at $y_{m}$ which can be bounded by

$$
0 \leq y_{m} \leq \sqrt{\sqrt{4 x^{2}-\eta+\eta^{2} / 4}-x^{2}-1+\eta / 2},
$$

where $\eta$ is defined above and $a$ can be chosen as

$$
a= \begin{cases}10 & \text { for } b=3 \\ 5 & \text { for } b \geq 4\end{cases}
$$

We have plotted in Fig. 2 the spectral radii $\varrho\left(K_{P, 1}(x+i y)\right)$ (solid lines) and $\varrho\left(K_{P, 1}^{1}(x+i y)\right)$ (dashed-dotted lines) against $y$ for $N=64, b=32$ and for fixed $x=2, x=2.5$ and $x=3$. We can see that $\varrho\left(K_{P, 1}(x+i y)\right)$ attains its maximum for $y_{m} \neq 0$ and $\varrho\left(K_{P, 1}^{1}(x+i y)\right)$ seems to attain its maximum for $y_{m}=0$. 


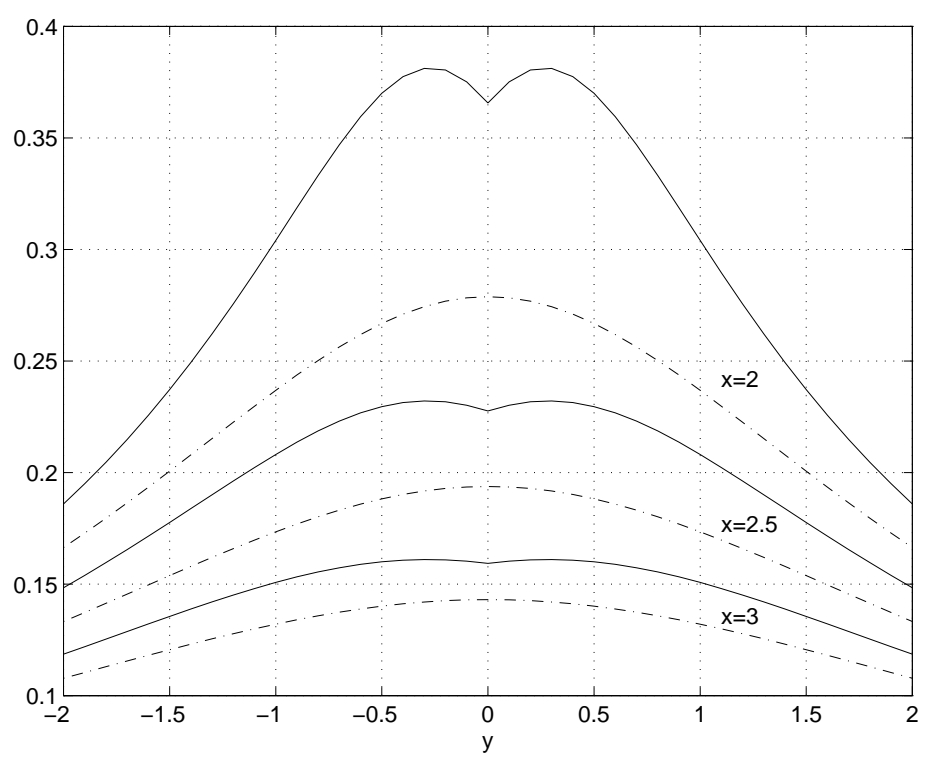

Fig. 2. Spectral radii $\varrho\left(K_{P, 1}(x+i y)\right)$ (solid lines) and $\varrho\left(K_{P, 1}^{1}(x+i y)\right)$ (dashed-dotted lines) for the one-dimensional heat equation

4. Abscissa of $\omega$-convergence. Leimkuhler [4] introduced the notion of abscissa of $\omega$-convergence $\xi_{\omega}$ defined by

$$
\xi_{\omega}=\inf \left\{\xi: \varrho_{\xi}(\mathcal{K})<\omega\right\},
$$

where $\mathcal{K}$ is the waveform relaxation operator. He demonstrated that $\left|\mathcal{K}^{n} f\right|_{\widehat{T}_{\omega}}$ is approximately bounded by $e \omega^{n}|f|_{\widehat{T}_{\omega}}$, where $\widehat{T}_{\omega}=1 / \xi_{\omega}$ is interpreted as the size of the window of $\omega$-convergence and $|\cdot|_{\widehat{T}_{\omega}}$ is the uniform norm in the space of continuous functions defined on $\left[0, \widehat{T}_{\omega}\right]$. The above estimate is rather crude and it was demonstrated in [3] that $\widehat{T}_{\omega}=4 / \xi_{\omega}$ better approximates the size of the window of $\omega$-convergence than the heuristic estimate proposed in [4]. (Compare also the discussion in Section 2.) We will use the more accurate estimate $\widehat{T}_{\omega}=4 / \xi_{\omega}$ in the remainder of this section.

In the Laplace transform domain the formula for $\xi_{\omega}$ takes the form

$$
\xi_{\omega}=\inf \{x: \sup \{\varrho(K(s))<\omega: \operatorname{Re}(s)=x\}\} .
$$

In this section we will find upper bounds on $\xi_{\omega}$ for $K(s)=K_{P, 1}^{1}(s)$ and $K(s)=K_{P, 1}(s)$ given by (2.4) and (2.3), respectively. We have

$$
\varrho\left(K_{P, 1}^{1}(s)\right) \leq \frac{2\left|\delta_{1}\right|}{x^{2}+y^{2}}<\frac{2\left|\delta_{1}\right|}{x^{2}}
$$

for $s=x+i y$. Hence,

$$
\xi_{\omega} \leq \sqrt{a / \omega}
$$


where $a$ is defined by (3.1). This translates into the bound on the window of $\omega$-convergence of the form

$$
\widehat{T}_{\omega} \geq 4 \sqrt{a / \omega} .
$$

For block size $b \geq 3$ and $K(s)=K_{P, 1}(s)$ we obtain

$$
\varrho\left(K_{P, 1}(s)\right) \leq \frac{a\left(x^{2}+y^{2}\right)}{\left(x^{2}-1\right)^{2}+2\left(x^{2}+1\right) y^{2}+y^{4}},
$$

where $a$ is given by (3.2). This implies

$$
\varrho\left(K_{P, 1}(s)\right) \leq \frac{a x^{2}}{\left(x^{2}-1\right)^{2}} \quad \text { and } \quad \xi_{\omega} \leq \frac{1}{2}(\sqrt{a / \omega}+\sqrt{a / \omega+4}) .
$$

Hence,

$$
\widehat{T}_{\omega} \geq \frac{8 \sqrt{\omega}}{\sqrt{a}+\sqrt{a+4 \omega}} .
$$

In the case of $(2.5)$ with $N=64$ and $b=32$ we obtain for $\omega=1$ the inequalities

$$
\begin{array}{ll}
\widehat{T}_{1} \geq 2.83 & \text { using (4.1), and } \\
\widehat{T}_{1} \geq 1.53 & \text { using (4.2). }
\end{array}
$$

The first inequality is too optimistic while the second one is in quite a good agreement with numerical results presented in [2]. We recall that using Fig. 1 the corresponding estimates were found to be $\widehat{T}_{1} \approx 2.3$ and $\widehat{T}_{1} \approx 2.0$, respectively.

5. Semidiscretization of the heat equation in two space variables. Semidiscretization of the heat equation in two space variables leads to the linear system of the form (2.5) with

$$
Q=\left[\begin{array}{ccccc}
T & -I & & & \\
-I & T & -I & & \\
& \ddots & \ddots & \ddots & \\
& & -I & T & -I \\
& & & -I & T
\end{array}\right] \in \mathbb{R}^{N^{2} \times N^{2}},
$$

where $I$ is the identity matrix of dimension $N \times N$ and $T$ is defined by

$$
T=\left[\begin{array}{ccccc}
4 & -1 & & & \\
-1 & 4 & -1 & & \\
& \ddots & \ddots & \ddots & \\
& & -1 & 4 & -1 \\
& & & -1 & 4
\end{array}\right] \in \mathbb{R}^{N \times N} .
$$


We consider the splitting of the matrix $Q$ corresponding to the block GaussJacobi method with the additional requirement that the block size is a multiple of $N$. This means that $Q=M+D$ where

$$
M=\operatorname{diag}(\underbrace{\widehat{M}, \ldots, \widehat{M}}_{s \text { times }})
$$

and

$$
\widehat{M}=\left[\begin{array}{ccccc}
T & -I & & & \\
-I & T & -I & & \\
& \ddots & \ddots & \ddots & \\
& & -I & T & -I \\
& & & -I & T
\end{array}\right] \in \mathbb{R}^{N b \times N b}
$$

with $b s=N$ for some integers $b$ and $s$. Then it is easy to verify that the corresponding matrix $K_{P, r}(s)$ can again be expressed by (2.6) for $b \geq 3$ and by (2.7) for $b=2$, where $\Delta_{1}, \Delta_{2}, \Gamma_{1}$ and $\Gamma_{2}$ are defined as in Section 2 but with ones replaced by $I$ 's and with zeros replaced by zero matrices of dimension $N \times N$. As a consequence all the results of Sections 2-4 are also applicable to the waveform relaxation methods approximating the linear system corresponding to the heat equation in two space variables.

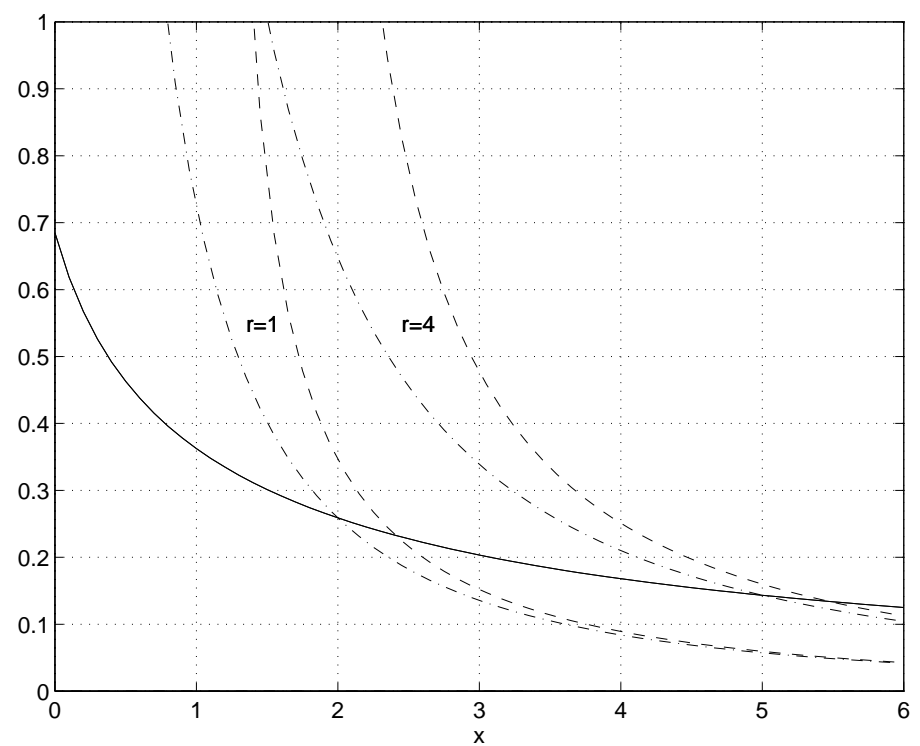

Fig. 3. Spectral radii corresponding to the two-dimensional heat equation. Dashed lines: $\sup \left\{\varrho\left(K_{P, r}(s)\right): \operatorname{Re}(s)=x\right\} ;$ dashed-dotted lines: $\sup \left\{\varrho\left(K_{P, r}^{1}(s)\right): \operatorname{Re}(s)=x\right\}$; solid line: $\sup \left\{\varrho\left(K_{N}(s)\right): \operatorname{Re}(s)=x\right\}$

We have plotted in Fig. 3 the maxima of the spectral radii $\varrho\left(K_{P, r}(s)\right)$ and $\varrho\left(K_{P, r}^{1}(s)\right)$ for fixed $x=\operatorname{Re}(s)$ and $r=1$ and $r=4$. We have also 


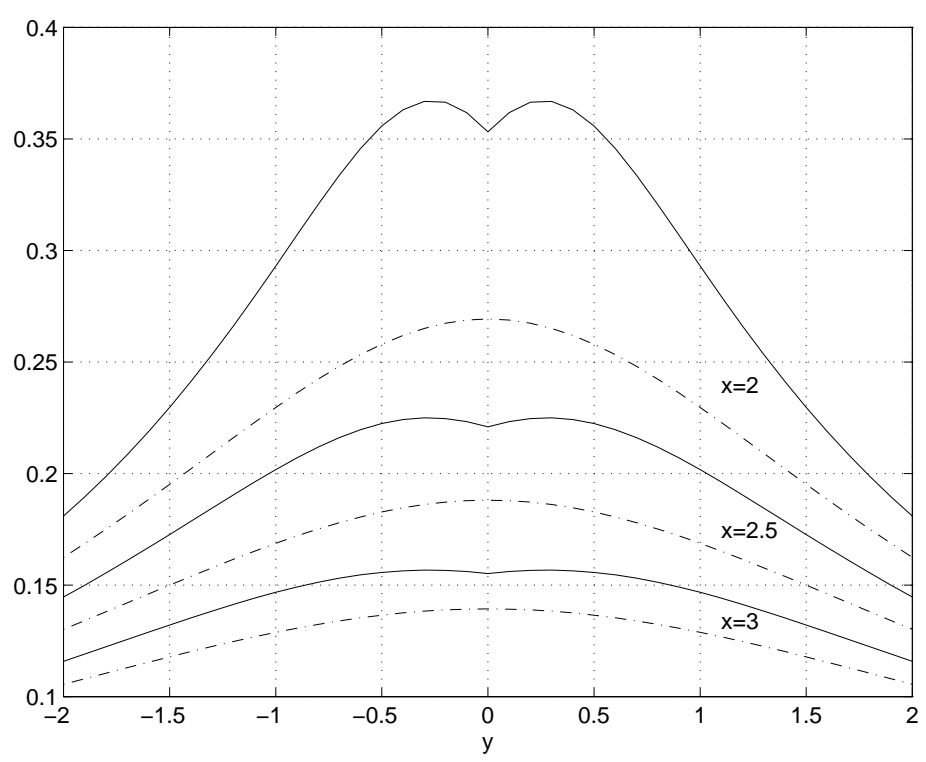

Fig. 4. Spectral radii $\varrho\left(K_{P, 1}(x+i y)\right)$ (solid lines) and $\varrho\left(K_{P, 1}^{1}(x+i y)\right)$ (dashed-dotted lines) for the two-dimensional heat equation

plotted in Fig. 4 the spectral radii $\varrho\left(K_{P, 1}(x+i y)\right)$ and $\varrho\left(K_{P, 1}^{1}(x+i y)\right)$ as functions of $y$ for $x=2, x=2.5$ and $x=3$. The graphs in Figs. 3 and 4 correspond to $N=8\left(N^{2}=64\right)$ and $b=4(N b=32)$. These figures are analogues of Figs. 1 and 2 corresponding to the one-dimensional case. The spectral radii in Fig. 3 corresponding to preconditioned waveform relaxation iterations do not differ much from the corresponding radii in Fig. 1 for the one-dimensional case. The difference between these radii is somewhat more pronounced in Figs. 2 and 4 because of the different scale on the vertical axes. We can see again that the analysis of convergence of preconditioned waveform relaxation iterations based on $K_{P, r}(s)$ gives more accurate estimates of the window of $\omega$-convergence that the analysis based on $K_{P, r}^{1}(s)$ which was employed in [2].

6. Concluding remarks. Error analysis of preconditioned waveform relaxation iterations for linear systems of differential equations is performed. This analysis is carried out in the Laplace transform domain and takes into account all terms in the corresponding error equation. Closed form expressions are obtained for the Laplace transform $K_{P, r}(s)$ of the kernel of the waveform relaxation operator with initial error of the form $e^{(0)}(t)=t^{r} v$, where $v$ is an arbitrary vector of appropriate dimension. These expressions lead to the more accurate estimates of the window of $\omega$-convergence than those in [2], which were based on the first term only in the expansion for 
$K_{P, r}(s)$. The new estimates are in quite a good agreement with the numerical results for linear systems resulting from semidiscretization of the heat equation in one and two dimensions.

Figs. 1 and 3 also illustrate that the convergence is slower for greater $p$. We conclude that preconditioning is the most effective for the first few iterations.

\section{References}

[1] K. Burrage, Parallel and Sequential Methods for Ordinary Differential Equations, Oxford Univ. Press, Oxford, 1995.

[2] K. Burrage, Z. Jackiewicz, S. P. Nørsett and R. Renaut, Preconditioning waveform relaxation iterations for differential systems, BIT 36 (1996), 54-76.

[3] Z. Jackiewicz, B. Owren and B. D. Welfert, Pseudospectra of waveform relaxation operators, Computers Math. Appl. 36 (1998), 67-85.

[4] B. Leimkuhler, Estimating waveform relaxation convergence, SIAM J. Sci. Comput. 14 (1993), 872-889.

[5] E. Lelerasmee, A. Ruehli and A. Sangiovanni-Vincentelli, The waveform relaxation method for time domain analysis of large scale integrated circuits, IEEE Trans. on CAD of IC and Systems 1 (1982), 131-145.

[6] U. Miekkala and O. Nevanlinna, Convergence of dynamic iteration methods for initial value problems, SIAM J. Sci. Statist. Comput. 8 (1987), 459-482.

[7] O. Nevanlinna, Remarks on Picard-Lindelöf iteration, Part I, BIT 29 (1989), $328-346$.

Andrzej Augustynowicz

Institute of Mathematics

University of Gdańsk

Wita Stwosza 57

80-952 Gdańsk, Poland

E-mail: antek@ksinet.univ.gda.pl
Zdzisław Jackiewicz Department of Mathematics Arizona State University Tempe, Arizona 85287

$$
\text { U.S.A. }
$$

E-mail: jackiewi@math.la.asu.edu 